\title{
The influence of thickness on ammonia gas sensitivity of reduced graphene oxide films
}

\author{
Tran Quang Nguyen ${ }^{1}$, Huynh Tran My Hoa ${ }^{2}$, Tran Quang Trung ${ }^{2, *}$
}

${ }^{1}$ University Information Technology, VNU-HCM

${ }^{2}$ Department of Solid State Physics, Faculty of Physics, University of Science, VNU-HCM

\section{Correspondence}

Tran Quang Trung, Department of Solid State Physics, Faculty of Physics, University of Science, VNU-HCM

Email: trungvicr@yahoo.com.sg

\section{History}

- Received: 2018-12-08

- Accepted: 2019-04-22

- Published: 2019-08-04

DOI :

https://doi.org/10.32508/stdj.v22i3.1236

\section{Check for updates}

\section{Copyright}

(c) VNU-HCM Press. This is an openaccess article distributed under the terms of the Creative Commons Attribution 4.0 International license.

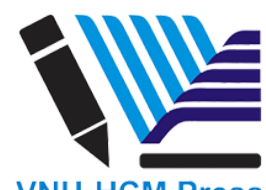

\begin{abstract}
Graphene is a single carbon layer in a two-dimensional (2D) lattice. Its delocalized $\pi$ bonds give rise to unique electronic properties, but these $\pi$ bonds are easily influenced by the environment. Meanwhile, many publications present that the sensitivity of graphene is not only necessarily intrinsic to this material but also by external defect. In this study, we produced reduced Graphene Oxide ( $\mathrm{rGO}$ ) sensors based on random $\mathrm{rGO}$ plates. We analyzed the ammonia $\left(\mathrm{NH}_{3}\right)$ sensitivity of such sensors as a function of thickness of rGO films (in terms of change in transparence) at room temperature. When the thickness of $\mathrm{rGO}$ films decreased, a maximum response was observed for the thinnest rGO film (the transparence was $84 \%$ ), with a sensitivity up to $38 \%$. Our results suggest that the dependence of $\mathrm{NH}_{3}$ sensitivity on $\mathrm{rGO}$ films thickness is dictated by the fully exposed surface area for thinnest films and by 2D charge carrier hopping through edge defects.

Key words: Graphene, Ammonia gas sensing, Reduced Graphene Oxide, Defects
\end{abstract}

\section{INTRODUCTION}

Many researchers have shown that the sensitivity of rGO film can be decreased by oxygen-containing groups (epoxy groups, hydroxyl groups, etc.) ${ }^{1,2}$, and by surface and edge defects of $\mathrm{rGO}^{3,4}$. The effects of the oxygen-containing groups on the gas-sensing signal can be controlled by the reduction process from GO to rGO films (dependent on the reducing agent). Moreover, as reported by Lili Liu et al. ${ }^{3}$, structural defects can also affect gas sensitivity signals. When the defects are in the rGO lattice, they will naturally have impacts on the electronic structures, such as bond lengths in the strain fields of the defects, the local rehybridization of sigma and $\pi$-orbitals, and the scattering of electron waves ${ }^{3}$.

In this study, we investigated ammonia $\left(\mathrm{NH}_{3}\right)$ gas sensitivity with different thickness of rGO films by two steps. Firstly, rGO films were synthesized by the chemical method with different thickness through different volumes of rGO solution ${ }^{5}$, and secondly, these $\mathrm{rGO}$ films were investigated for $\mathrm{NH}_{3}$ gas sensitivity at room temperature ${ }^{6}$. It is important to note that the effect of the oxygen-containing groups on the sensitivity of rGO films was fixed by the stable reducing condition. In the study herein, we focus on the structural defects ( surface and edge defects) that directly affect gas sensitive signals when the rGO films are overlapped. These defects can be controlled by the different thicknesses of rGO films because the electronic properties of two-dimensional (2D) lattices strongly depend on the thickness of materials $3,5,7$.

\section{METHODS}

\section{Synthesis of the reduced graphene oxide (rGO) and fabrication of gas sensor}

The fabrication process of gas sensor based on the reduced graphene oxide (rGO) material was performed by the following protocol. Firstly, the graphite (Sigma-Aldrich, India) was exfoliated by microwave irradiation and then, the exfoliation graphite was oxidized to GO by chemical method- with the mixture of $0.8 \mathrm{~g} \mathrm{KMnO}_{4} / 16 \mathrm{ml} \mathrm{H} \mathrm{PO}_{4} / 0.1 \mathrm{~g} \mathrm{NaNO} 3$ (modified Hummers method): $\mathrm{KMnO}_{4}$ (Duc Giang Detergent Chemicals JSC, Vietnam), $\mathrm{H}_{3} \mathrm{PO}_{4}$ (Xilong Scientific Co., Ltd, China), and $\mathrm{NaNO}_{3}{ }^{1,8}$. Secondly, GO material was deposited directly on spaced inter-digitated silver electrodes patterned on the clean $\left(1 \mathrm{~cm}^{2}\right)$ substrate by using spin coating method (Figure 1a). During this period, we used different volumes of GO solution (from $0.04 \mathrm{ml}$ to $0.25 \mathrm{ml}$ ) with the aim of changing the thickness of the achieved rGO films. Then, these GO films were exposed with hydrazine agent at $80^{\circ} \mathrm{C}$ and heated quickly at $350^{\circ} \mathrm{C}$ to reduce GO films to rGO films. Finally, we investigated the $\mathrm{NH}_{3}$ gas sensitivity as a function of the thickness of rGO films at room temperature. Additionally, we used different spaced inter-digitated silver electrodes (space between lines was $1 \mathrm{~mm}$ and $1.5 \mathrm{~mm}$ ) (Figure 1b). 


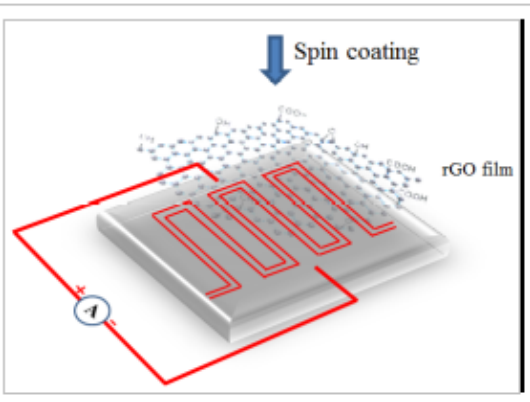

(a)

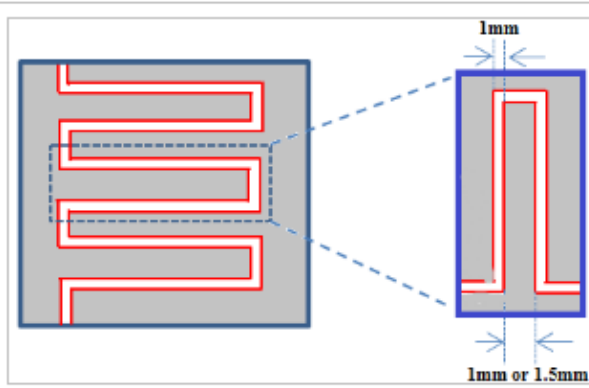

(b)

Figure 1: The gas sensor. (a) The spaced inter-digitated substrate with rGO film ; (b) space between lines was 1 $\mathrm{mm}$ and $1.5 \mathrm{~mm}$.

Based on the rGO films used, we had two sensing samples which were named "rGO- space -volume". For example, rGO-1.0-0.04ml sample was fabricated on 1.0 $\mathrm{mm}$ spaced inter-digitated silver electrodes with 0.04 $\mathrm{ml}$ of GO solution. Tell what the $2^{\text {nd }}$ sensing sample was.

\section{The measurement system}

The gas sensor was connected to two probes in the test chamber and the signal was displayed on the screen computer by the transducer through the LABVIEW software. The measurement consisted of two processes that were called absorption and desorption. In the absorption process, the $\mathrm{NH}_{3}$ gas flowed into the test chamber for the period time and the change in resistance of sensor was recorded during that time ${ }^{2}$. In the desorption process, the argon (Ar) gas was pumped into the test chamber to re-establish the initial resistance of $\mathrm{rGO}^{2}$.

\section{RESULTS}

\section{Investigating the change of thickness of rGO films}

Caterina Soldano et al. ${ }^{6}$ showed that graphite crystal becomes highly transparent when thinned down to a graphene monolayer (using Chemical Vapor Deposition method). Indeed, in the visible light region, the transparency of graphene monolayer was $97.7 \%$ and it decreased linearly when the thickness of graphene was increased to five layers. However, as the thickness of graphene film continually increased, the transparency of graphene film should decrease nonlinearly ${ }^{6,9}$.

Herein, we investigate the different thickness of rGO films using the transparency spectra by ultravioletvisible (UV-vis) and Stylus method, as described in
Figure 2.

\section{Interaction of ammonia gas with the rGO films}

After preparation of the gas sensor, we measured $\mathrm{NH}_{3}$ gas sensitivity $(\Delta R / R 0)$ of rGO films. For the spaced inter-digitated silver electrodes of $1.5 \mathrm{~mm}$ (i.e. rGO1.5 sample), as shown in Figure 3a, the thinnest rGO film (rGO-1.5-0.04ml) demonstrated the highest sensitivity (34\%).

When the volume of the GO solution was increased from $0.04 \mathrm{ml}$ to $0.25 \mathrm{ml}$, the sensitivity decreased from $34 \%$ to $4.5 \%$ (Figure $3 \mathbf{b}$ ).

The result of rGO-1.0 in Figure 4a was similar to the result of rGO-1.5 in Figure 3a. When the volume of GO solution was increased, the thickness of rGO films became thicker and the sensing signal of rGO films decreased (Figure 4b). However, from Figure 3a and b, it can be seen that the $\mathrm{NH}_{3}$ gas sensitivity of rGO1.0 (38\%) was higher than that of rGO-1.5 (34\%). Comparing our experimental results with the results of other research groups on the gas sensitivity of twodimensional (2D) materials, there was some similarity. Therefore, the gas sensitive signals of $2 \mathrm{D}$ materials are optimal when their thickness are decreased to monolayer ${ }^{5,7}$.

\section{DISCUSSION}

By ultraviolet-visible (UV-vis) setting, when the volume of GO solution was increased in the range of 0.04 $\mathrm{ml}$ to $0.25 \mathrm{ml}$ (Figure 2a), the transparency of rGO films was decreased in the range of $84 \%$ (rGO-0.04 $\mathrm{ml}$ sample) to $74 \%$ (rGO- $0.25 \mathrm{ml}$ sample) at $\lambda=550$ $\mathrm{nm}$, as shown in Figure $\mathbf{2 b}$. The result of the transparency of the rGO films was similar with the variation of thickness from $151 \mathrm{~nm}$ to $784 \mathrm{~nm}$ (Figure 2b), 


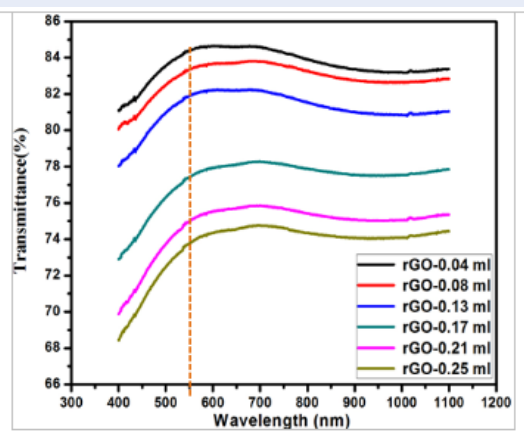

(a)

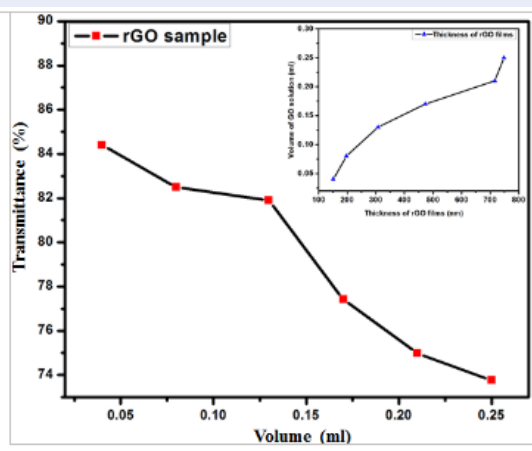

(b)

Figure 2: The transparency spectra. (a) the different thickness of $\mathrm{rGO}$ films, (b) dependence of transmittance on the $\mathrm{GO}$ volume (at $\lambda=550$ ). In the inset: the different thickness of $\mathrm{rGO}$ film on $\mathrm{GO}$ volume.

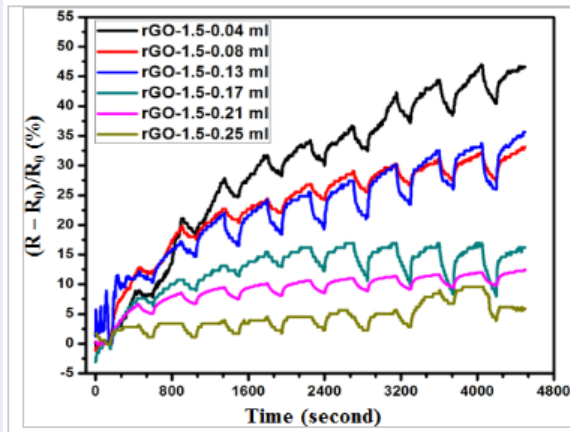

(a)

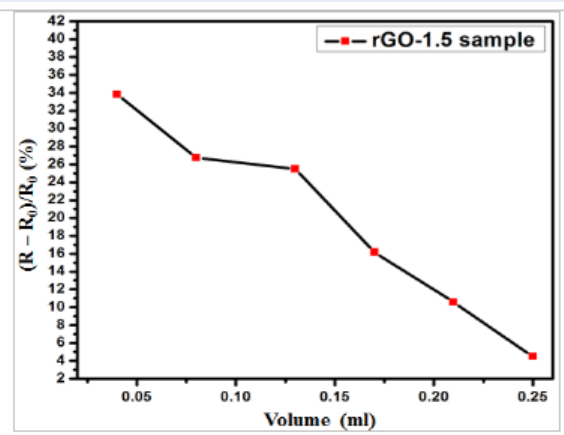

(b)

Figure 3: The characteristic of $\mathrm{NH}_{3}$ gas sensitivity. (a) the $\Delta \mathrm{R} / \mathrm{R} 0$ value of $\mathrm{rGO}-1.5$, (b) the $\Delta R / R 0$ value with different rGO-1.5 volume.

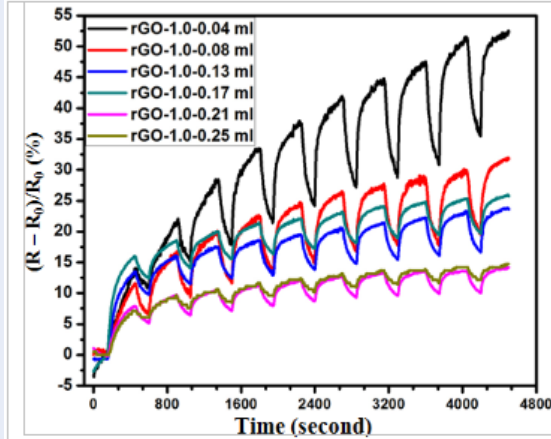

(a)

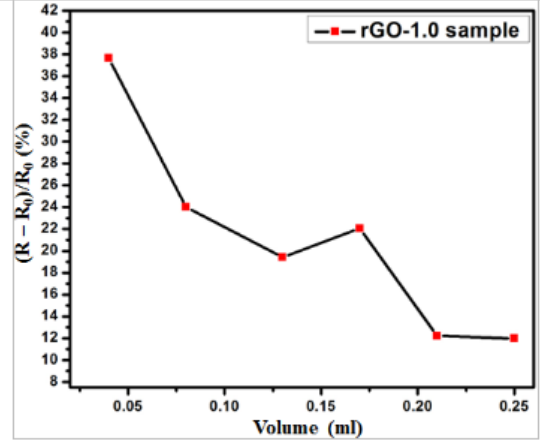

(b)

Figure 4: The characteristic of $\mathrm{NH}_{3}$ gas sensitivity. (a) the $\Delta R / R 0$ value of rGO-1.0, (b) the $\Delta R / R 0$ value with different rGO-1.0 volume. 
by the Stylus method. These results demonstrate that the transparency of rGO films is strongly affected by the thickness of the rGO film.

The transport of electrons of the gas sensor- based on rGO material- directly affected the sensitivity signal $(\Delta \mathrm{R} / \mathrm{R} 0)$ of the device. In Figure 3, when the rGO film was thinner, its gas sensitivity increased. This result could be explained by the fact that the rGO sheets in the rGO film were arranged in the most uniform manner and there was less overlap in the thinnest rGO film ${ }^{3}$. This produced the convenience for interaction between the $\mathrm{NH}_{3}$ gas molecules and rGO sheets, not only on the planar sheet but also on the edge defects $^{3,4}$. Hence, the surface resistance of the rGO film changed significantly.

This problem could be overcome by reducing the thickness of the rGO film and the distance between the electrode lines. In Figure 4, the sensitivity signal of this device was improved (from 34\% to 38\%). This can be explained by the fact that as the space between inter-digitated silver electrodes were decreased, the electron trajectories were shorter. This was easy for transmitting sensing signals to the measurement equipment. From our results, we suggest that when the space between electrode lines is continually decreased to micrometers, one rGO sheet can be used for making gas sensor and the response signal of the devices can be made more optimal.

\section{CONCLUSION}

When the rGO film was thinner, its gas sensitivity increased remarkably as follows: the rGO film decreased 5-fold, and the response signal of the device increased 3.2-fold. At that time, the distance between electrode lines decreased 1.5-fold, and the response signal increased $\sim 1.2$ times. However, our study has also shown the limitations of the thickness film; we fabricated the gas sensor substrate with a large electrode distance (millimeter). Moreover, we deposited the rGO film by chemical method which led to the
rGO sheets being dispersed non-uniformly and overlapping together. In future experimental studies, we will decrease the electrode distance to yield the lowest rGO sheets, and the thickness of rGO films would be made thinner.

\section{COMPETING INTERESTS}

No conflict of interest declared.

\section{AUTHORS' CONTRIBUTIONS}

Tran Quang Nguyen implemented the experiment about the fabrication of gas sensor and the investigation of ammonia $\left(\mathrm{NH}_{3}\right)$ gas sensitivity based on reduced graphene oxide (rGO). Huynh Tran My Hoa synthesized rGO material from graphite flakes. We proposed the experiment plan and wrote the manuscript together. Tran Quang Trung helped us evaluated the stability of ammonia $\left(\mathrm{NH}_{3}\right)$ gas sensitivity based on rGO films.

\section{ACKNOWLEDGMENTS}

We would like to acknowledge Department of Solid State Physics, Faculty of Physics, University of Science, VNU-HCM for fruitful discussion. This research is funded by University Information Technology (VNU-HCM) under grant number D1-2019-11.

\section{REFERENCES}

1. Tang S, Cao Z. J Phys Chem C. 2012;116:8778-8791.

2. Gao X, Jang J, Nagase S. J Phys Chem C. 2010;114:832-842.

3. Liu L, Qing M, Wang Y, Chen S. Journal of Materials Science \& Technology. 2015;31:599e606.

4. Acik M, Yves J. Japanese Journal of Applied Physics. 2011;50:070101.

5. Salehi-Khojin A, Kevin DE, Lin Y, Ran K, Haasch RT, Zuo JM, et al. Applies Physics Letters. 2012;100:033111.

6. Ceterina, Mahmood A, Dujardin E. Erik Dujardin, ScienceDirect, carbon. 2010;48:2127-2150.

7. Cui S, Haihui P, Wells SA, Wen Z, Mao S, Chang J, et al. Nature Commun. 2016;10.

8. Prezioso S, Perrozzi F, Giancaterini L, Cantalini C, Treossi $\mathrm{E}_{\iota}$ Palermo V, et al. J Phys Chem C. 2013;117:10683-10690.

9. Blake P, Brimicombe PD, Nair RR, Booth TJ, Jiang D. Nano Letters. 2008;8:1704-8. 